\title{
Health-related Quality of Life Outcomes of Adalimumab for Patients with Early Rheumatoid Arthritis: Results from a Randomized Multicenter Study
}

\author{
VIBEKE STRAND, ANNE M. RENTZ, MARY A. CIFALDI, NAIJUN CHEN, SANJOY ROY, and DENNIS REVICKI
}

\begin{abstract}
Objective. Rheumatoid arthritis (RA) is associated with significant impairments in health-related quality of life (HRQOL). We evaluated patient-reported outcomes including HRQOL outcomes following adalimumab plus methotrexate (MTX) therapy in patients with early RA.

Methods. PREMIER was a phase III, multicenter, randomized, double-blind, active-comparator clinical trial in early RA. Patients aged $\geq 18$ years were randomly assigned to receive adalimumab $40 \mathrm{mg}$ every other week (eow) plus weekly MTX, weekly MTX, or adalimumab $40 \mathrm{mg}$ eow for 104 weeks. American College of Rheumatology (ACR) criteria were used to evaluate clinical efficacy and response. Outcomes were assessed using the Health Assessment Questionnaire Disability Index (HAQ-DI), Short-Form 36 Health Survey (SF-36), Short-Form 6 Dimension (SF-6D), visual analog scale (VAS) assessments of global disease activity (patient's global assessment; PtGA) and pain, Functional Assessment of Chronic Illness Therapy-Fatigue (FACIT-F), and Health Utility Index Mark 3 (HUI-3). Results. Of 799 patients enrolled, 268 received adalimumab plus MTX, 257 received MTX monotherapy, and 274 received adalimumab monotherapy. Patients treated with adalimumab plus MTX demonstrated significant baseline to Week 104 improvements in HAQ-DI $(\mathrm{p}<0.0001)$, SF-36 Physical Component Summary $(\mathrm{p}<0.0001), 4$ SF-36 domains [physical function $(\mathrm{p}<0.0001)$, bodily pain $(\mathrm{p}<$ $0.0001)$, vitality $(\mathrm{p}=0.0139)$, role limitations-physical $(\mathrm{p}=0.0005)]$, SF-6D $(\mathrm{p}=0.0152)$, VAS-PtGA $(p<0.0001)$, VAS-pain $(p<0.0001)$, FACIT-F $(p<0.0001)$, and HUI-3 ( $p=0.0034)$ scores versus patients treated with MTX monotherapy. Both SF-6D and HUI-3 were found to be sensitive preference-based measures for assessing the effects of treatment on multidimensional function. No clinically meaningful differences between adalimumab and MTX monotherapy groups were observed for most measures. For each measure, there was significant association between HRQOL improvement and ACR clinical response.

Conclusion. Adalimumab plus MTX significantly improved physical functioning and HRQOL in patients with early RA over 2 years of treatment. (ClinicalTrials.gov identifier NCT00195663). (First Release Nov 1 2011; J Rheumatol 2012;39:63-72; doi:10.3899/jrheum.101161)
\end{abstract}

Key Indexing Terms:

RHEUMATOID ARTHRITIS ADALIMUMAB

PATIENT-REPORTED OUTCOMES

\author{
HEALTH-RELATED QUALITY OF LIFE \\ PREFERENCE-BASED MEASURES
}

From the Division of Immunology/Rheumatology, Stanford University School of Medicine, Portola Valley, California, USA; Center for Health Outcomes Research, United BioSource Corporation, Bethesda, Maryland, USA; and Abbott Laboratories, Abbott Park, Illinois, USA.

Funded by Abbott Laboratories, Abbott Park, IL, USA. V. Strand has served as a consultant to Abbott Laboratories.

$V$. Strand, MD, Division of Immunology/Rheumatology, Stanford University School of Medicine; A.M. Rentz, MSPH; D. Revicki, PhD, Center for Health Outcomes Research, United BioSource Corporation; M.A. Cifaldi, PhD, RPh, MSHA; N. Chen, MS; S. Roy, MS, Abbott Laboratories.

Address correspondence to Dr. V. Strand, Division of

Immunology/Rheumatology, Stanford University School of Medicine, 306 Ramona Road, Portola Valley, CA 94028, USA.

E-mail:vstrand@stanford.edu

Full Release Article. For details see Reprints/Permissions at jrheum.org Accepted for publication August 4, 2011.
Patient-reported outcomes (PRO) provide important assessments of functioning and well-being from the patient's perspective $^{1,2}$. Rather than simply serving as a complement to clinical (physician-reported) measures, PRO have been shown to better discriminate active treatment from placebo in randomized controlled trials in rheumatoid arthritis (RA) $)^{3,4,5,6,7,8,9}$. RA significantly affects health-related quality of life (HRQOL), including physical functioning, pain, fatigue and vitality, emotional and social well-being, and work productivity $1,3,10,11,12,13$. Preference-based HRQOL measures, such as the Short-Form 6 Dimension (SF-6D) derived from the Medical Outcomes Study Short-Form 36 Health Survey $(\mathrm{SF}-36)^{14}$ and the Health Utilities Index Mark 3 (HUI-3) ${ }^{15}$, contribute to our understanding of the influence of RA and 
treatment-associated improvements on health outcomes and quality-adjusted life-years, thereby assisting physicians and patients in making therapeutic decisions ${ }^{16}$.

Adalimumab is a human anti-tumor necrosis factor monoclonal antibody with demonstrated efficacy in patients with $\mathrm{RA}^{17,18}$, psoriatic $\operatorname{arthritis}^{19}$, ankylosing spondylitis ${ }^{20}$, Crohn's disease ${ }^{21}$, plaque psoriasis ${ }^{22}$, and juvenile idiopathic arthritis $^{23}$. The PREMIER trial demonstrated that the combination of adalimumab plus methotrexate (MTX) was well tolerated and more effective than either monotherapy in treating patients with early RA ${ }^{18}$. The objective of these analyses was to examine the effect of these treatments on HRQOL in this trial, comparing the relative sensitivity of SF-6D and HUI-3 measured during the trial.

\section{MATERIALS AND METHODS}

Study sample. PREMIER was a 2-year, randomized, double-blind, active comparator-controlled, phase III clinical trial conducted at 133 sites in North America, Europe, and Australia ${ }^{18}$. MTX-naive patients $\geq 18$ years of age with active RA ( $\geq 8$ swollen joints, $\geq 10$ tender joints, and an erythrocyte sedimentation rate $\geq 28 \mathrm{~mm} / \mathrm{h}$ or C-reactive protein concentration $\geq 1.5 \mathrm{mg} / \mathrm{dl}$, in addition to rheumatoid factor positivity or $\geq 1$ joint erosion) and disease duration $<3$ years were randomized to receive adalimumab $40 \mathrm{mg}$ subcutaneously every other week plus weekly oral MTX, adalimumab monotherapy, or MTX monotherapy. Institutional review boards at participating centers approved the protocol. All patients provided written informed consent. Results of this trial have been published ${ }^{18}$. (ClinicalTrials.gov identifier NCT00195663)

Baseline assessments. Demographic and clinical characteristics included age, sex, disease duration, prior use of disease-modifying antirheumatic drugs (DMARD), concomitant use of corticosteroid, all components of the American College of Rheumatology (ACR) response criteria, and 28-joint Disease Activity Score (DAS28).

Clinical and PRO measures. ACR50 response and radiographic data at Week 52 were the co-primary outcome measures; secondary outcomes included ACR 20/50/70/90 responses, radiographic data, and physical function and HRQOL data at Week $104^{18}$. Planned exploratory analyses of the relationship between clinical responses and HRQOL outcomes categorized patients as ACR50 responder or nonresponder status based on data from Weeks 12 to 104.

PRO included 3 components of the ACR response criteria: Health Assessment Questionnaire Disability Index (HAQ-DI ${ }^{24}$ ), visual analog scale (VAS) assessments of global disease activity (patient's global assessment; PtGA) and pain, and the Functional Assessment of Chronic Illness Therapy-Fatigue (FACIT-F). These measures are well validated in $\mathrm{RA}^{25,26,27,28,29,30}$. FACIT-F measures fatigue and its effect on functioning and daily activities over the previous 7 days $^{28}$; scores range from 0 (none) to 52 (greater fatigue). A change of -0.22 in HAQ-DI and 4 points in FACIT-F are considered minimum clinically important differences (MCID) ${ }^{30}$. Normative values for HAQ-DI in the general population are $\leq 0.5^{24,31}$.

HRQOL measures included SF-36 and HUI-3. Data were collected at baseline and Weeks 12, 26, 52, 76, and 104. SF-6D scores were calculated based on the SF-36. SF-36 is a generic HRQOL instrument that consists of 8 domains (physical function, bodily pain, role limitations-physical, general health, vitality, social function, role limitations-emotional, and mental health) scored from 0 to 100 , with higher scores indicating better health status, and Physical (PCS) and Mental Component Summary (MCS) scores normed to the general population with a mean of 50.0 and an SD of $10.0^{32}$. Norm-based scores can also be calculated for the SF-36 domains, similar to the summary scores, with a mean of 50.0 and SD of 10.0 (higher scores indicating better health status). Changes of 5.0 to 10.0 points in domain scores and 2.5 to 3.0 points in PCS and MCS scores represent MCID $3,10,12,13,33,34,35$. SF-36 has demonstrated reliability and validity $3,10,32$ and responsiveness to change in patients with $\mathrm{RA}^{10,33}$. SF-6D is a preference-based generic health measure derived from the SF-36 ${ }^{14}$. SF-6D scores were calculated directly based on selected items from the SF-36. A change of 0.03 is considered the $\mathrm{MCID}^{36,37}$. SF-6D has demonstrated construct validity and responsiveness for use in RA $^{10,36,37,38}$

The HUI-3 is a collection of preference-based HRQOL measures consisting of 8 self-administered items assessing functional capacity in 8 domains: vision, hearing, speech, ambulation, dexterity, emotion, cognition, and pain, over the previous 4 weeks ${ }^{39,40}$. Index scores range from 0 to 1.0 , with higher scores representing better health status. A change of $\geq 0.03$ points is considered the MCID ${ }^{40}$. The HUI-3 has good reliability and construct validity $15,39,41$ and has been used in clinical studies in RA ${ }^{10}$.

Statistical analyses. Primary HRQOL endpoints were a priori identified as baseline to Week 104 mean changes in HAQ-DI and SF-36 PCS and MCS scores, following the precedent set by the US Food and Drug Administration for labeling for "improvement and maintenance of physical function and HRQOL." Secondary endpoints included patient VAS (PtGA and pain), SF-36 domain, SF-6D, FACIT-F, and HUI-3 scores over 104 weeks. Analyses were conducted using the intention-to-treat population, defined as all randomized patients who received $\geq 1$ treatment dose. Analyses compared adalimumab plus MTX versus MTX monotherapy and adalimumab monotherapy versus MTX monotherapy. All analyses included all observed data.

Baseline demographics, clinical characteristics, and HRQOL measures were summarized using descriptive statistics. Chi-square tests and Student t tests were performed to examine baseline differences between treatment groups.

For repeated-measurement variables, a mixed model with random intercept was applied to compare mean HRQOL scores between the treatment groups ${ }^{42}$. Adjusted mean scores are reported. The mixed model included terms for treatment group, week, treatment-by-week interaction, and relevant baseline score as covariates. Statistical analyses of HAQ-DI, PtGA, pain, SF-36 summary and domain scores, and FACIT-F were predefined; SF-6D and HUI-3 were considered supportive and exploratory. Spydergrams were used to compare mean SF-36 domain scores between the adalimumab plus MTX and MTX monotherapy groups ${ }^{43}$. For these analyses, the SF-36 domain scores for baseline, 52-week, and 104-week visits were included.

An exploratory data analysis evaluated the relationship between ACR50 response status and changes in HRQOL measures. Analysis of covariance models were used to estimate least-squares mean baseline to Week 104 change scores for HAQ-DI, SF-36 PCS and MCS, SF-6D, FACIT-F, and HUI-3 scores. The models included factors for ACR50 response status, age, sex, and relevant baseline scores.

\section{RESULTS}

A total of 799 patients participated in our study: 268 in the adalimumab plus MTX group, 257 in the MTX monotherapy group, and 274 in adalimumab monotherapy group. Baseline demographic and clinical characteristics were similar between the 3 treatment groups (Table 1). Mean duration of RA at baseline was 9 months. There were no statistically significant differences in baseline PRO scores between the combination and MTX monotherapy groups. The adalimumab and MTX monotherapy groups differed significantly in several PRO measures (HAQ-DI, SF-36 PCS, SF-36 physical function domain, SF-36 social function domain, SF-6D, FACIT-F, and HUI-3), which favored MTX monotherapy (Table 1).

Primary HRQOL endpoints Adalimumab combination therapy vs MTX monotherapy. 
Table 1. Patient demographics, disease characteristics, and patient-reported outcome scores at baseline. Data are mean (SD) unless otherwise noted.

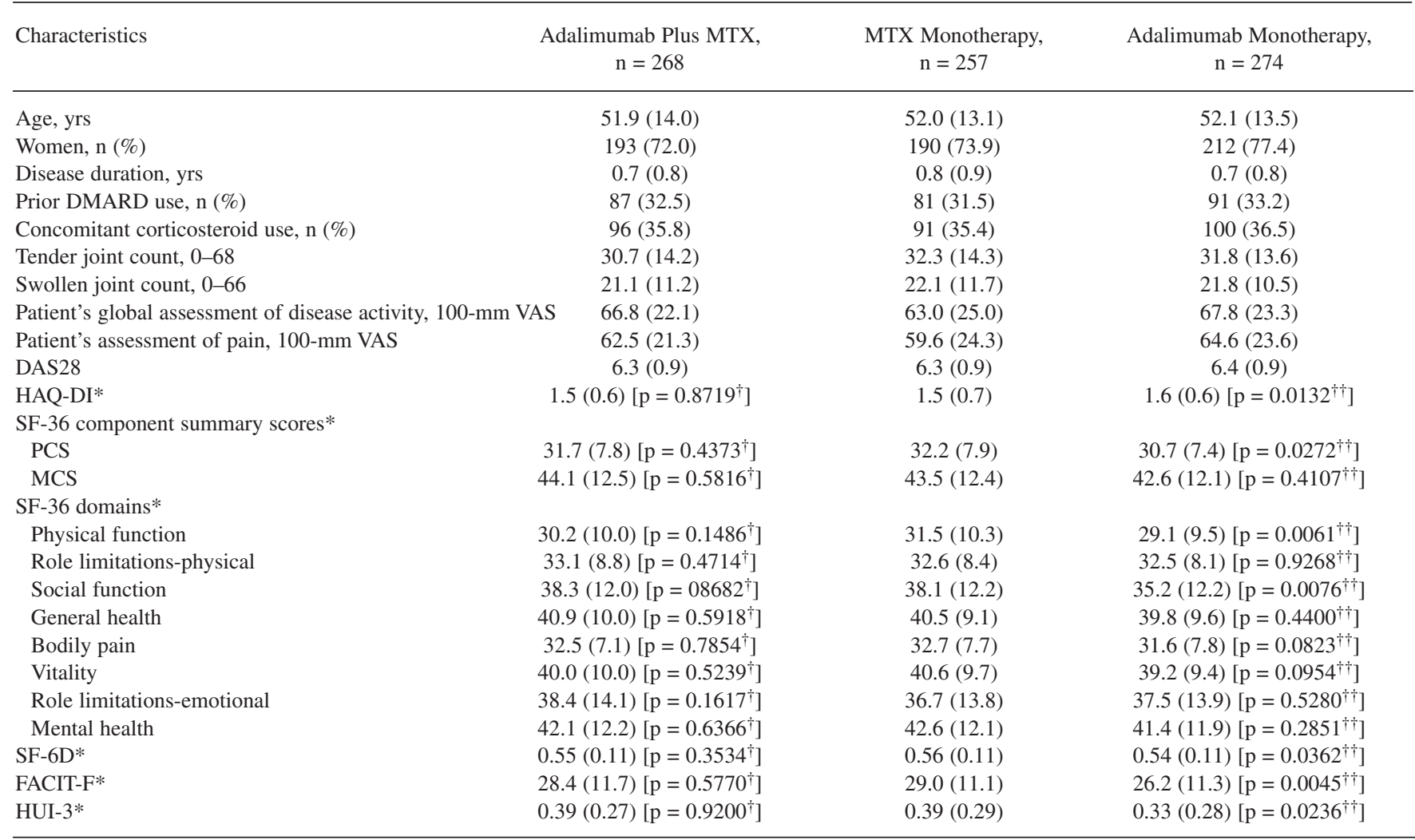

* Unadjusted mean (SD). ${ }^{\dagger} \mathrm{p}$ values from Student $\mathrm{t}$ tests between MTX monotherapy and adalimumab plus MTX combination therapy. ${ }^{\dagger \dagger} \mathrm{p}$ values from Student $\mathrm{t}$ tests between MTX and adalimumab monotherapy groups. DAS28: 28-joint Disease Activity Score; DMARD: disease-modifying antirheumatic drug; FACIT-F: Functional Assessment of Chronic Illness Therapy-Fatigue; HAQ-DI: Health Assessment Questionnaire Disability Index; HUI-3: Health Utility Index Mark 3; MCS: mental component summary; MTX: methotrexate; PCS: physical component summary; SF-6D: Short-Form 6 Dimension; SF-36: Short-Form 36 Health Survey; VAS: visual analog scale.

Patients treated with adalimumab plus MTX reported statistically significant baseline to Week 104 improvements in HAQ-DI scores versus patients treated with MTX monotherapy. There were significant main effects of treatment $(\mathrm{p}<$ $0.0001)$ and significant treatment-by-week interactions for HAQ-DI scores $(\mathrm{p}<0.0001$; Table 2$)$. Patients treated with adalimumab plus MTX reported early and sustained decreases (improvements) over the first 52 weeks of treatment and maintained values less than US general population norms for the remainder of the study (Figure 1). The MTX monotherapy group reported less early improvement in mean HAQ-DI scores; this difference was sustained over the study duration. Significantly more patients receiving combination therapy reported clinically meaningful improvements in physical functioning (MCID criterion of $\geq 0.22$ points) at Week 104 ( $67.5 \%$ vs $58.7 \%$, respectively; $p=0.037$ ).

Statistically significant improvements from baseline to Week 104 in SF-36 PCS scores but not in SF-36 MCS scores were observed with adalimumab plus MTX versus MTX monotherapy. There were significant main effects of treatment ( $p<0.0001$ ) but no significant treatment-by-week interactions for SF-36 PCS scores (Table 2). More patients receiving com- bination therapy reported clinically meaningful improvements in SF-36 PCS scores (MCID criterion of $\geq 3.0$ points) at Week 104 (56.4\% vs 43.6\%; $\mathrm{p}=0.0469)$.

Adalimumab monotherapy vs MTX monotherapy. Patients in both monotherapy groups reported improvements in HAQ-DI scores. There was a significant treatment-by-week interaction ( $\mathrm{p}<0.0001$ ) for HAQ-DI scores, but main effects of treatment were nonsignificant (Table 2). Although the adalimumab monotherapy group had more impaired physical function at baseline, the trajectory of mean HAQ-DI scores over the study duration was comparable between the monotherapy groups (Figure 1).

No significant differences were observed between the monotherapy groups in SF-36 PCS scores (Table 2). However, more patients receiving adalimumab monotherapy than those receiving MTX monotherapy reported clinically meaningful improvements in SF-PCS scores (MCID criterion of $\geq 3.0$ points) at Week 104 (52.4\% vs $47.6 \%$, respectively; p = 0.0292). For SF-36 MCS scores, there were significant main effects of treatment $(\mathrm{p}=0.0148)$ without significant treatment-by-week interactions (Table 2). The MTX monotherapy group reported a slightly greater mean change from baseline 
Table 2. Mean patient-reported outcome scores by treatment group and study visit. Data are adjusted mean (SD).

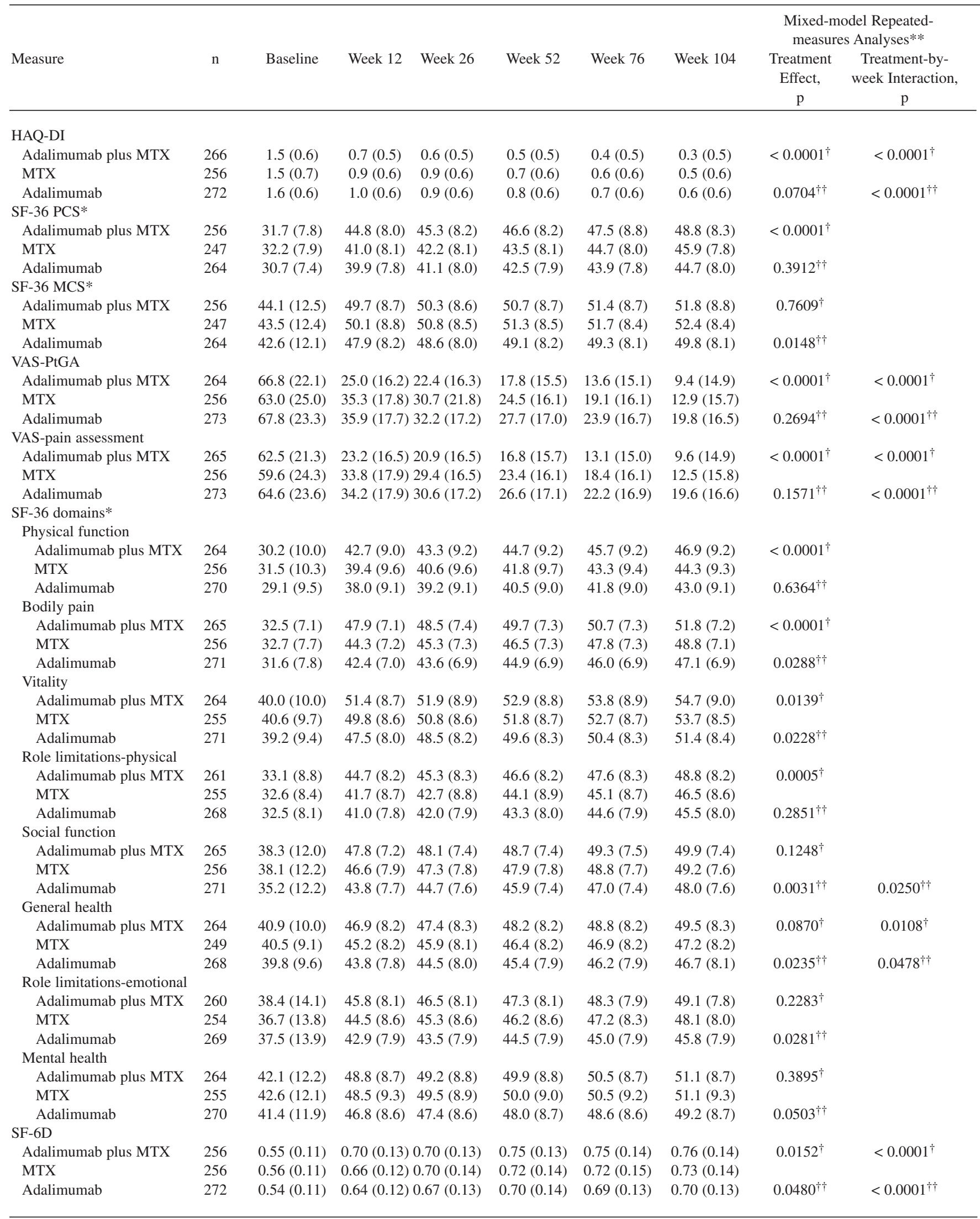




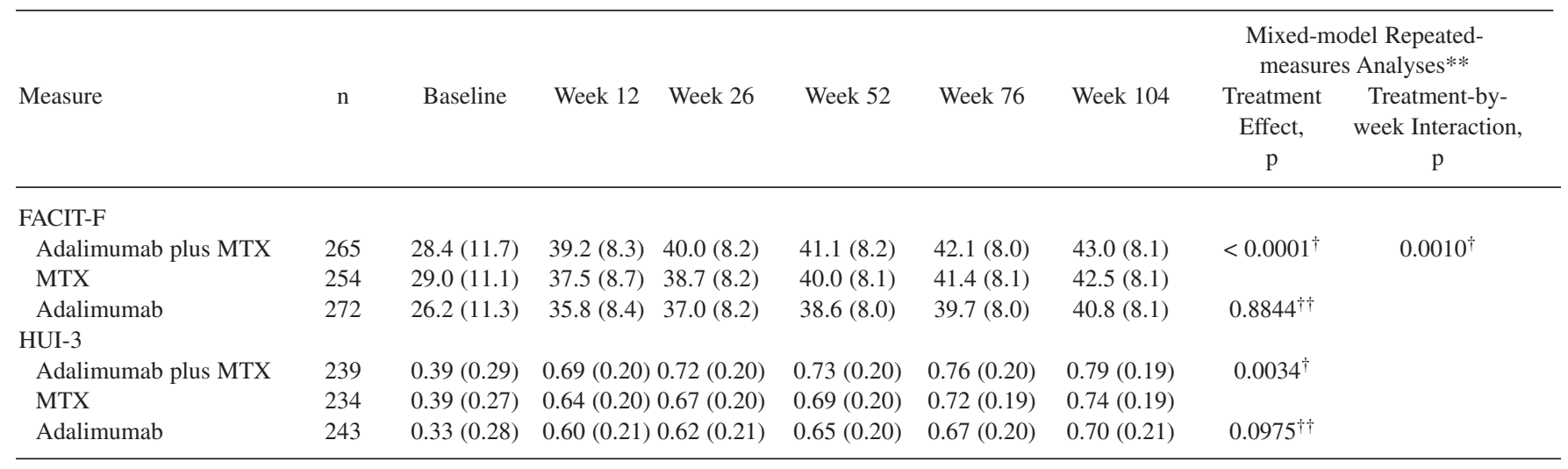

* Normative scores (mean 50.0, SD 10.0), with greater scores indicating better health status. ** Two-tailed p values from repeated-measures random-intercept model, including treatment, week, treatment-by-week interaction, and baseline score as independent variables. The interaction term was retained in the model when the $\mathrm{p}$ value for the interaction was $<0.10$. Otherwise, the interaction term was removed. ${ }^{\dagger}$ Adalimumab plus MTX monotherapy vs MTX monotherapy. ${ }^{\dagger \dagger}$ Adalimumab monotherapy vs MTX monotherapy. FACIT-F: Functional Assessment of Chronic Illness Therapy-Fatigue; HAQ-DI: Health Assessment Questionnaire Disability Index; HUI-3: Health Utility Index Mark 3; MCS: mental component summary; MTX: methotrexate; PCS: physical component summary; SF-6D: Short-Form 6 Dimension; SF-36: Short-Form 36 Health Survey; VAS: visual analog scale.

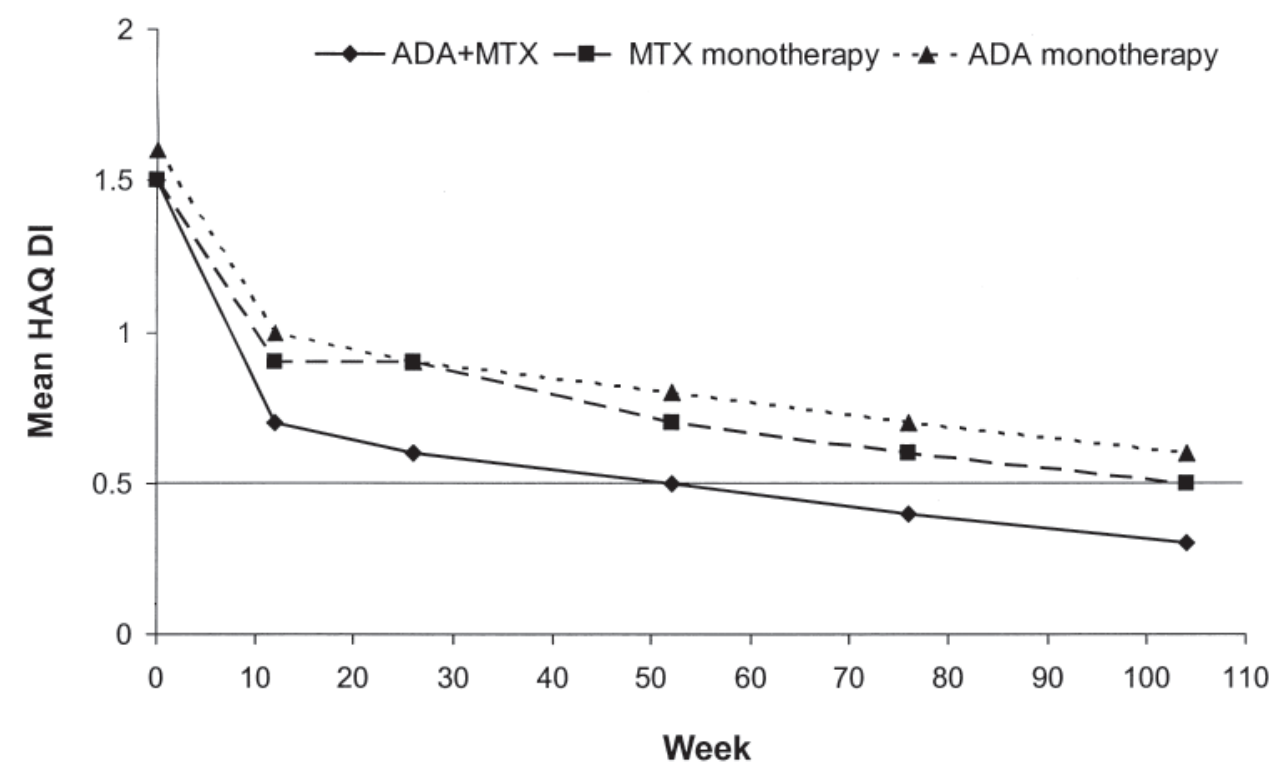

Figure 1. Mean Health Assessment Questionnaire Disability Index (HAQ-DI) scores by treatment group and study visit. Horizontal line at 0.49 is the reported mean HAQ-DI for the general population ${ }^{24}$. ADA: adalimumab; MTX: methotrexate.

to Week 104 in SF-36 MCS scores versus the adalimumab monotherapy group ( 8.8 vs 7.2 points), but this difference was not clinically meaningful.

Secondary HRQOL endpoints

Adalimumab combination therapy vs MTX monotherapy. Treatment with adalimumab plus MTX was associated with significant main treatment effects $(\mathrm{p}<0.0001)$ and treatment-by-week interactions $(p<0.001)$ for VAS PtGA and pain assessments (Table 2). Significant main effects of treatment were also observed for 4 SF-36 domain scores (physical function, bodily pain, vitality, and role limitations-physical), and a significant treatment-by-week interaction was observed for general health (Table 2).

Figure 2 summarizes the mean SF-36 domain scores between the combination treatment and MTX monotherapy groups. The largest differences were observed between the treatment groups on physical function, bodily pain, vitality, and role limitations-physical scores, all favoring the combination therapy group.

In addition, treatment with adalimumab plus MTX was associated with significant main treatment effects for SF-6D 


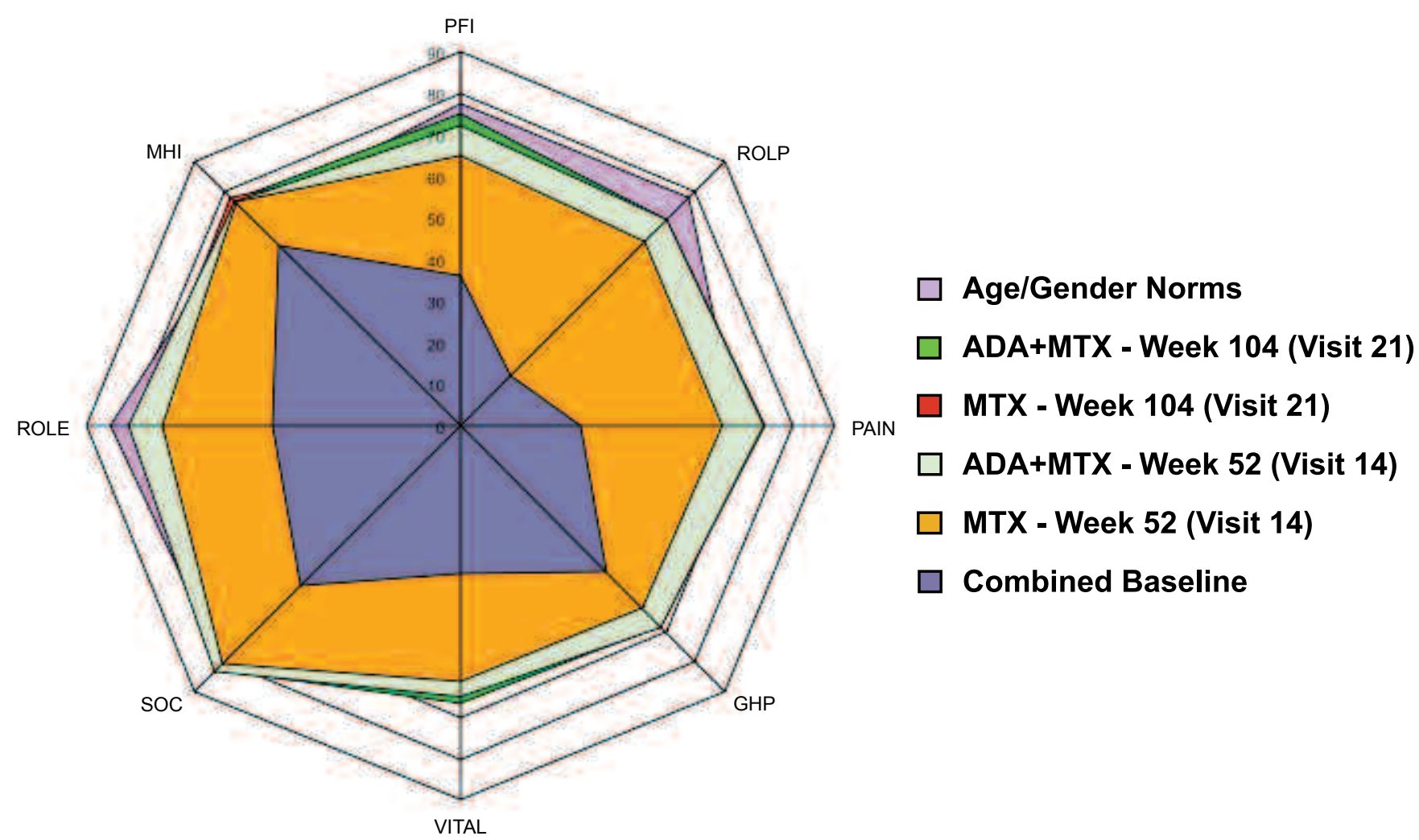

Figure 2. Spydergram summarizing mean Short-Form 36 Health Survey (SF-36) domain scores for the adalimumab (ADA) plus methotrexate (MTX) and MTX monotherapy groups at baseline, Week 52, and Week 104, compared with age-/sex-matched norms. Norm-based domain scores were converted to a scale of 0-100, consistent with other publications showing spydergrams in rheumatoid arthritis ${ }^{43}$. PFI: physical function, ROLP: role limitations-physical, PAIN: bodily pain, GHP: general health, VITAL: vitality, SOC: social function, ROLE: role limitations-emotional, and MHI: mental health.

$(\mathrm{p}=0.0152)$, FACIT-F $(\mathrm{p}<0.0001)$, and HUI-3 $(\mathrm{p}=0.0034)$ scores and treatment-by-week interactions for SF-6D $(\mathrm{p}<$ $0.0001)$ and FACIT-F $(\mathrm{p}=0.001)$ scores (Table 2). Mean improvements were comparable across preference-based measures (SF-6D, HUI-3) for patients treated with adalimumab plus MTX (Figure 3A) and patients treated with MTX (Figure 3B).

Adalimumab monotherapy vs MTX monotherapy. Significant treatment-by-week interactions without main treatment effects were observed for VAS assessments (Table 2). There were significant main effects of treatment for 5 SF-36 domain scores (bodily pain, vitality, social function, general health, and role limitations-emotional) and significant treatment-by-week interactions for social function and general health (Table 2). The observed improvements in SF-36 domain scores favored the MTX monotherapy group, but differences were generally small.

There was a significant main effect of treatment for derived SF-6D $(p=0.0480)$ and a significant treatment-by-week interaction $(\mathrm{p}<0.0001)$ for the SF-6D (Table 2). No significant differences between the monotherapy groups were observed on the FACIT-F or HUI-3 (Table 2). Mean improvements were comparable across preference-based measures for both monotherapy groups (Figures 3B, 3C).
Exploratory analysis: relationship between clinical response and PRO. Exploratory analyses examined the relationship between ACR response criteria and baseline to Week 104 changes in HAQ-DI, SF-36 PCS and MCS, SF-6D, FACIT-F, and HUI-3 scores after adjustment for age, sex, and baseline score. Mean change scores were significantly improved in ACR50 responders versus nonresponders on each measure $(\mathrm{p}<0.0001$; Table 3).

\section{DISCUSSION}

The PREMIER trial demonstrated the clinical efficacy and safety of adalimumab plus MTX in patients with early RA ${ }^{18}$. The current analyses demonstrated that these responses were associated with significant, clinically meaningful improvements in HRQOL and preference-based measures.

At study entry, patients reported significant impairments in physical function and HRQOL compared with the US general population. Baseline mean HAQ-DI scores were 1.5 to 1.6 for patients in PREMIER compared with the reported mean HAQ-DI score of 0.49 for the general population ${ }^{24}$. Over the 2-year study, mean HAQ-DI scores improved to 0.3 (adalimumab plus MTX group), 0.5 (MTX monotherapy group), and 0.6 (adalimumab monotherapy group). Twelve-week improvements in HAQ-DI were significantly greater in the

Personal non-commercial use only. The Journal of Rheumatology Copyright $\odot$ 2012. All rights reserved. 

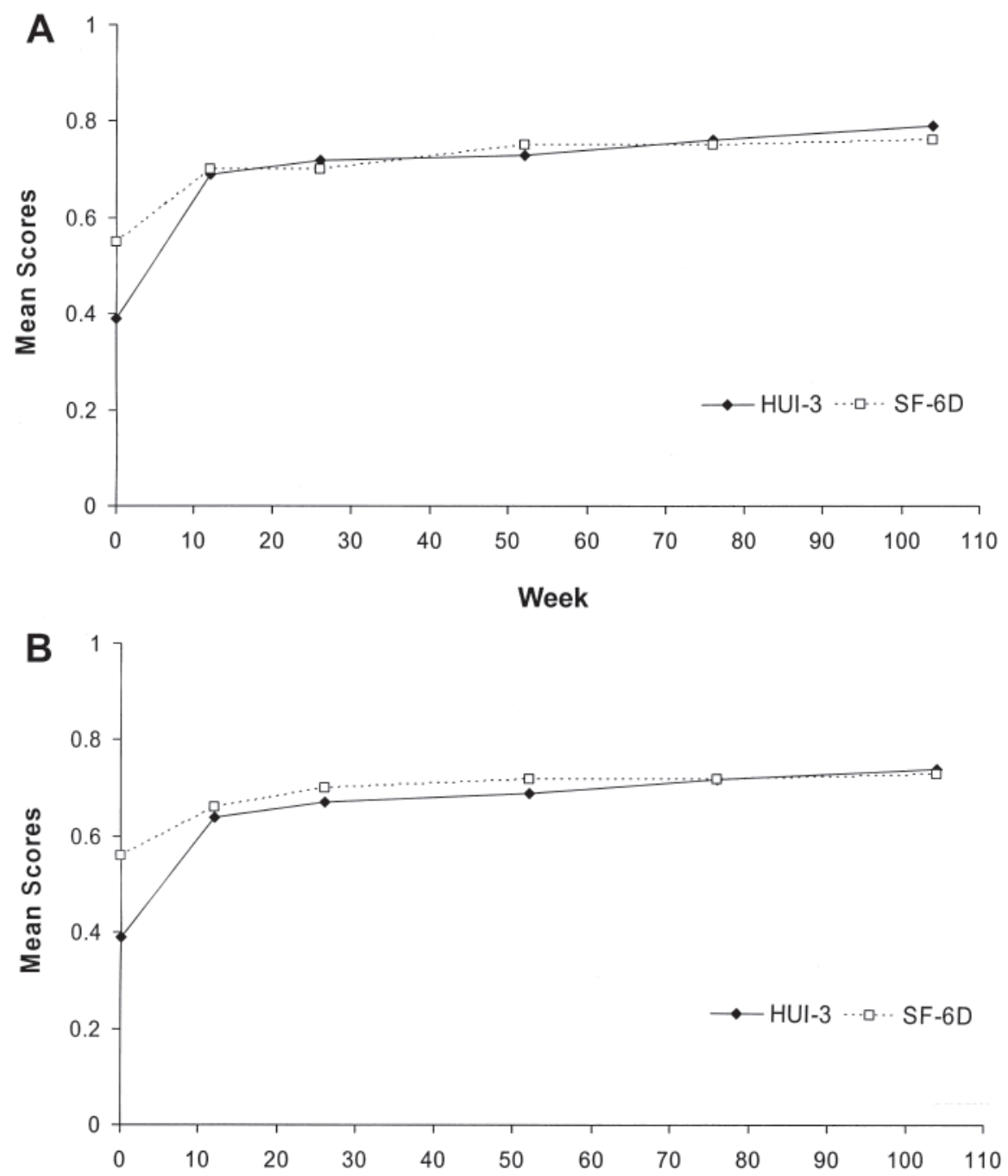

Week

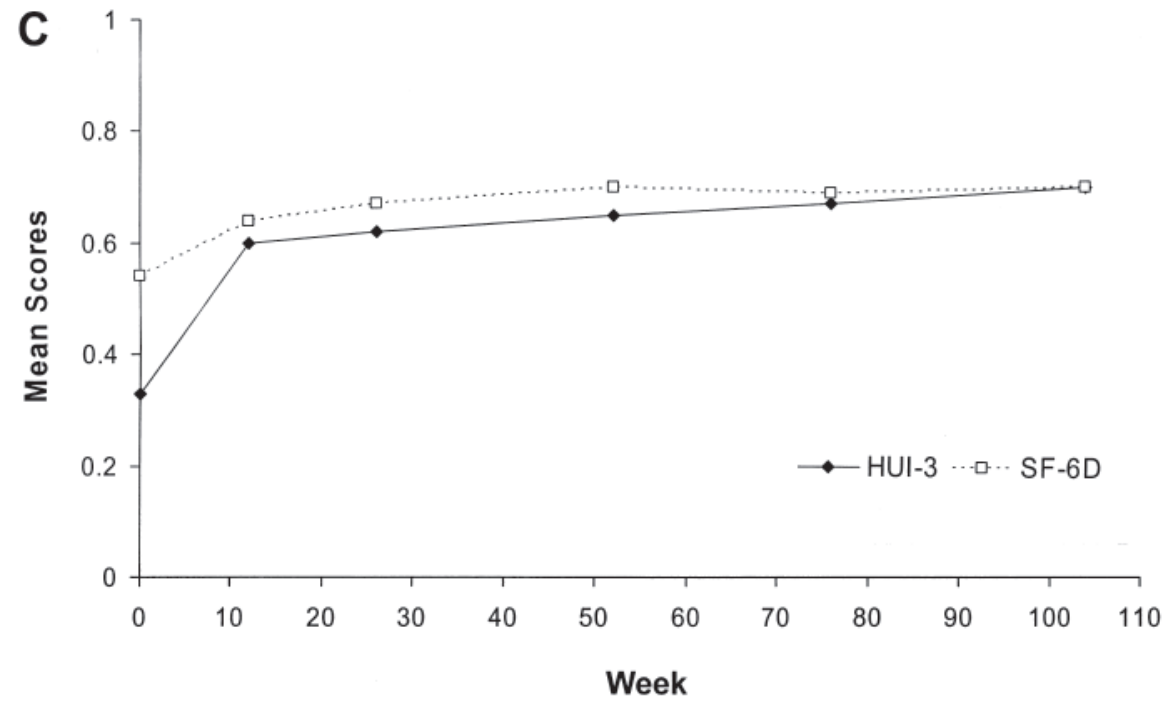

Figure 3. Mean Health Utility Index Mark 3 (HUI-3) and Short-Form 6 Dimension (SF-6D) scores by treatment group and study visit. (A) Adalimumab plus methotrexate (MTX). (B) MTX monotherapy. (C) Adalimumab monotherapy. 
Table 3. Association of ACR50 response with mean changes from baseline to Week 104 in patient-reported outcome scores.

\begin{tabular}{|c|c|c|c|}
\hline \multirow[b]{2}{*}{ Measure } & \multicolumn{3}{|c|}{$\begin{array}{c}\text { ACR50 Improvement Status, adjusted mean (SE) for } \\
\text { change scores }\end{array}$} \\
\hline & $\begin{array}{l}\text { Nonresponder, } \\
\mathrm{n}=169\end{array}$ & $\begin{array}{l}\text { ACR50 Responder, } \\
\qquad \mathrm{n}=369\end{array}$ & $\begin{array}{c}\text { Overall } \\
\mathrm{p}^{\dagger}\end{array}$ \\
\hline HAQ-DI & $-0.6(0.04)$ & $-1.2(0.03)$ & $<0.0001$ \\
\hline SF-36 PCS & $7.2(0.65)$ & $17.4(0.45)$ & $<0.0001$ \\
\hline SF-36 MCS & $4.7(0.81)$ & $8.8(0.56)$ & $<0.0001$ \\
\hline SF-6D & $0.10(0.01)$ & $0.22(0.01)$ & $<0.0001$ \\
\hline FACIT-F & $8.1(0.69)$ & $15.9(0.47)$ & $<0.0001$ \\
\hline HUI-3 & $0.22(0.02)$ & $0.41(0.01)$ & $<0.0001$ \\
\hline
\end{tabular}

\footnotetext{
$\dagger \mathrm{p}$ for overall test of differences between ACR50 responder groups from an analysis of covariance model that included ACR50 responder group, baseline score, age, and, sex. ACR50: American College of Rheumatology rating scale (50\% or more improvement); FACIT-F: Functional Assessment of Chronic Illness Therapy-Fatigue; HAQ-DI: Health Assessment Questionnaire Disability Index; HUI-3: Health Utility Index Mark 3: MCS: mental component summary; PCS: physical component summary; SF-6D: Short-Form 6 Dimension; SF-36: Short-Form 36 Health Survey.
}

adalimumab plus MTX group versus the MTX monotherapy group, and improvements were maintained over the study duration. Observed differences between treatment group means of 0.26 at Week 12 and 0.29 at Week 104 exceeded the a priori MCID for HAQ-DI ( 0.22 points $\left.{ }^{24}\right)$. Moreover, mean HAQ-DI scores in the combination therapy group were comparable to or better than the mean for the general population by Week 52, suggesting improvement from moderate to severe impairment in physical function to a level of performance of daily activities similar to that of the general population.

Baseline mean SF-36 PCS scores were 30.7 to 32.2 for patients in PREMIER compared with reported mean population-based scores of $50.0^{32}$ and 48.3 (age, sex, and race-adjusted score ${ }^{12}$ ). Over the 2-year study, mean SF-36 PCS scores improved to 48.8 (adalimumab plus MTX group), 45.9 (MTX monotherapy group), and 44.7 (adalimumab monotherapy group). Twelve-week improvements in SF-36 PCS were significantly greater in the adalimumab plus MTX group versus the MTX monotherapy group, and improvements were sustained over 2 years. Observed differences between treatment group means of 3.8 at Week 12 and 2.9 at Week 104 exceeded the a priori MCID criterion of 2.5 to 3.0 points $3,10,12,13,33,34,35$, indicating that combination therapy improves physical functioning in patients with early RA. The effects on physical functioning observed in our study are consistent with previous clinical trials of treatments for $\mathrm{RA}^{10}$.

These findings are further supported by improvements in SF-36 domain scores over time. Baseline scores for patients in PREMIER showed significant impairment across all domains versus age- and sex-matched norms specific to the protocol population, derived from the US population. Treatment-associated improvements over 2 years of adalimumab plus MTX treatment met the US normative values in 5 of 8 domains (pain, general health, vitality, social function, and mental health), compared with 3 of 8 domains with MTX monother- apy (vitality, social function, and mental health). In addition, there were statistically significant and clinically meaningful differences between adalimumab plus MTX and MTX monotherapy groups in SF-36 physical function, bodily pain, role limitations-physical, and vitality domain scores.

The adalimumab plus MTX group reported greater improvements on the 2 preference-based measures compared with the MTX group. SF-6D scores showed clinically meaningful improvements from baseline to Week 52 and Week 104 in both treatment groups. Differences between treatment group means at Week 104 were 0.03 for SF-6D, which met the MCID criteria of 0.03 for SF-6D ${ }^{36,37}$. For HUI-3 scores, the observed difference of 0.05 at Week 104 exceeded the a priori MCID criterion of 0.03 points $^{40}$. Based on our study, the directly measured HUI-3 and the SF-6D were sensitive preference-based measures for assessing the effects of RA treatments.

No clinically meaningful differences between the monotherapy groups were observed for most HRQOL measures. Although significant treatment-by-week differences were observed on the HAQ-DI, the adalimumab monotherapy group was more impaired at baseline, and the significant interaction indicates that the adalimumab group demonstrated a slightly increased rate of improvement in HAQ-DI scores (baseline to Week 104 changes: adalimumab, -1.03 points; MTX, -1.01 points). Baseline to Week 104 changes on SF-36 MCS scores were nearly comparable between groups (adalimumab, 7.2 points; MTX, 8.8 points) and differences were not clinically meaningful. These results suggest that adalimumab and MTX monotherapy may provide comparable improvements in HRQOL in patients with early RA.

For each HRQOL measure, there was a significant association between HRQOL improvement and clinical response, as assessed by the ACR50 response criteria. We observed significant improvements in HAQ-DI, SF-36 PCS, SF-36 MCS, SF-6D, FACIT-F, and HUI-3 scores, with the largest effects

Personal non-commercial use only. The Journal of Rheumatology Copyright @ 2012 . All rights reserved 
observed in physical health and functioning measures (HAQ-DI and SF-36 PCS scores). These findings support the clinical responsiveness of these PRO in patients with RA.

Limitations associated with HRQOL assessment in our study should be considered. First, one-third of patients in the MTX monotherapy group discontinued the study by Week 104. The use of observed data could have contributed to interaction effects because of discontinuation of a substantial number of patients from the MTX comparator group. However, because only observed data were used, the responder analyses results were not adversely affected. Moreover, the responder analyses may be conservative because $66 \%$ of the MTX monotherapy group was classified as ACR50 responders, whereas only $45 \%$ would have been considered responders if last-observation-carried-forward data had been used and only $43 \%$ would have been considered responders if data had been imputed. Second, because HRQOL endpoints are based on patient reports, it is unknown whether expectations for improvements in clinical and functional outcomes influenced the results. However, a consistent and significant relationship between clinical response levels and changes in HAQ-DI and SF-36 PCS scores was observed in our study, as reported ${ }^{3}$. A final consideration is that early and aggressive treatment for patients in PREMIER provided tight disease control, which may have contributed to the improvements in HRQOL observed in this patient population.

These results provide evidence supporting the statistically significant and clinically meaningful improvement in measures of physical functioning and preference-based HRQOL measures associated with adalimumab plus MTX treatment. In addition, patients treated with adalimumab plus MTX reported consistent and significant improvements across a range of HRQOL outcomes, and these improvements were maintained over a 2-year period. Together with previously reported findings on tolerability and clinical efficacy ${ }^{18}$, these results suggest that adalimumab plus MTX improves physical and multidimensional function or HRQOL and offers a comprehensive and effective therapy for patients with RA.

\section{ACKNOWLEDGMENT}

The authors acknowledge and thank Robin L. Stromberg, PhD, of Arbor Communications Inc., Ann Arbor, Michigan, and Michael A. Nissen, ELS, formerly of Abbott Laboratories, Abbott Park, Illinois, for editorial assistance in the development and revision of the manuscript.

\section{REFERENCES}

1. Mittendorf T, Dietz B, Sterz R, Cifaldi MA, Kupper H, von der Schulenburg JM. Personal and economic burden of late-stage rheumatoid arthritis among patients treated with adalimumab: an evaluation from a patient's perspective. Rheumatology 2008;47:188-93.

2. Pollard LC, Choy EH, Gonzalez J, Khoshaba B, Scott DL. Fatigue in rheumatoid arthritis reflects pain, not disease activity. Rheumatology 2006;45:885-9.

3. Tugwell P, Wells G, Strand V, Maetzel A, Bombardier C, Crawford B, et al. Clinical improvement as reflected in measures of function and health-related quality of life following treatment with leflunomide compared with methotrexate in patients with rheumatoid arthritis:
Sensitivity and relative efficiency to detect a treatment effect in a twelve-month, placebo-controlled trial. Leflunomide Rheumatoid Arthritis Investigators Group. Arthritis Rheum 2000;43:506-14.

4. Strand V, Cohen S, Crawford B, Smolen JS, Scott DL; Leflunomide Investigators Groups. Patient-reported outcomes better discriminate active treatment from placebo in randomized controlled trials in rheumatoid arthritis. Rheumatology 2004;43:640-7.

5. Cohen SB, Strand V, Aguilar D, Ofman JJ. Patient- versus physician-reported outcomes in rheumatoid arthritis patients treated with recombinant interleukin-1 receptor antagonist (anakinra) therapy. Rheumatology 2004;43:704-11.

6. Pincus T, Chung C, Segurado OG, Amara I, Koch GG. An index of patient reported outcomes (PRO-Index) discriminates effectively between active and control treatment in 4 clinical trials of adalimumab in rheumatoid arthritis. J Rheumatol 2006;33:2146-52.

7. Pincus T, Bergman MJ, Yazici Y, Hines P, Raghupathi K, Maclean R. An index of only patient-reported outcome measures, Routine Assessment of Patient Index Data 3 (RAPID3), in two abatacept clinical trials: Similar results to Disease Activity Score (DAS28) and other RAPID indices that include physician-reported measures. Rheumatology 2008;47:345-9.

8. Pincus T, Yazici Y, Bergman MJ. RAPID3, an index to assess and monitor patients with rheumatoid arthritis, without formal joint counts: Similar results to DAS28 and CDAI in clinical trials and clinical care. Rheum Dis Clin North Am 2009;35:773-8.

9. Pincus T, Swearingen CJ, Bergman MJ, Colglazier CL, Kaell AT, Kunath AM, et al. RAPID3 (Routine Assessment of Patient Index Data) on an MDHAQ (Multidimensional Health Assessment Questionnaire): Agreement with DAS28 (Disease Activity Score) and CDAI (Clinical Disease Activity Index) activity categories, scored in five versus more than ninety seconds. Arthritis Care Res 2010;62:181-9.

10. Strand V, Singh J. Newer biologic agents improve health related quality of life and productivity in rheumatoid arthritis. Drugs 2010;70:121-45.

11. Kvien TK. Epidemiology and burden of illness of rheumatoid arthritis. Pharmacoeconomics 2004;22 Suppl 1:1-12.

12. Kimel M, Cifaldi M, Chen N, Revicki D. Adalimumab plus methotrexate improved SF-36 scores and reduced the effect of rheumatoid arthritis (RA) on work activity for patients with early RA. J Rheumatol 2008;35:206-15.

13. Kosinski M, Zhao SZ, Dedhiya S, Osterhaus JT, Ware JE Jr. Determining minimally important changes in generic and disease-specific health-related quality of life questionnaires in clinical trials of rheumatoid arthritis. Arthritis Rheum 2000;43:1478-87.

14. Brazier J, Roberts J, Deverill M. The estimation of a preference-based measure of health from the SF-36. J Health Econ 2002;21:271-92.

15. Feeny DH, Furlong W, Torrance GW, Goldsmith CH, Zhu Z, DePauw $\mathrm{S}$, et al. Multiattribute and single-attribute utility functions for the Health Utilities Index Mark 3 system. Med Care 2002;40:113-28.

16. Revicki DA, Osoba D, Fairclough D, Barofsky I, Berzon R, Leidy NK, et al. Recommendations on health-related quality of life research to support labeling and promotional claims in the United States. Qual Life Res 2000;9:887-900.

17. Keystone EC, Kavanaugh AF, Sharp JT, Tannenbaum H, Hua Y, Teoh LS, et al. Radiographic, clinical, and functional outcomes of treatment with adalimumab (a human anti-tumor necrosis factor monoclonal antibody) in patients with active rheumatoid arthritis receiving concomitant methotrexate therapy: A randomized, placebo-controlled, 52-week trial. Arthritis Rheum 2004;50:1400-11.

18. Breedveld FC, Weisman MH, Kavanaugh AF, Cohen SB, Pavelka K, van Vollenhoven R, et al. The PREMIER study: A multicenter, randomized, double-blind clinical trial of combination therapy with adalimumab plus methotrexate versus methotrexate alone or adalimumab alone in patients with early, aggressive rheumatoid 
arthritis who had not had previous methotrexate treatment. Arthritis Rheum 2006;54:26-37.

19. Mease PJ, Gladman DD, Ritchlin CT, Ruderman EM, Steinfeld SD, Choy EH, et al; Adalimumab Effectiveness in Psoriatic Arthritis Trial Study Group. Adalimumab for the treatment of patients with moderately to severely active psoriatic arthritis: results of a double-blind, randomized, placebo-controlled trial. Arthritis Rheum 2005;52:3279-89.

20. van der Heijde D, Kivitz A, Schiff MH, Sieper J, Dijkmans BA, Braun J, et al; ATLAS Study Group. Efficacy and safety of adalimumab in patients with ankylosing spondylitis: results of a multicenter, randomized, double-blind, placebo-controlled trial. Arthritis Rheum 2006;54:2136-46.

21. Colombel JF, Sandborn WJ, Rutgeerts P, Enns R, Hanauer SB, Panaccione R, et al. Adalimumab for maintenance of clinical response and remission in patients with Crohn's disease: The CHARM trial. Gastroenterology 2007;132:52-65.

22. Saurat JH, Stingl G, Dubertret L, Papp K, Langley RG, Ortonne JP, et al; CHAMPION Study Investigators. Efficacy and safety results from the randomized controlled comparative study of adalimumab vs. methotrexate vs. placebo in patients with psoriasis (CHAMPION). Br J Dermatol 2008;158:558-66.

23. Lovell DJ, Ruperto N, Goodman S, Reiff A, Jung L, Jarosova K, et al; Pediatric Rheumatology Collaborative Study Group; Pediatric Rheumatology International Trials Organisation. Adalimumab with or without methotrexate in juvenile rheumatoid arthritis. N Engl J Med 2008;359:810-20.

24. Bruce B, Fries JF. The Stanford Health Assessment Questionnaire: Dimensions and practical applications. Health Qual Life Outcomes 2003;1:20.

25. Cole JC, Motivala SJ, Khanna D, Lee JY, Paulus HE, Irwin MR. Validation of single-factor structure and scoring protocol for the Health Assessment Questionnaire-Disability Index. Arthritis Rheum 2005;53:536-42.

26. Goekoop-Ruiterman YP, de Vries-Bouwstra JK, Allaart CF, van Zeben D, Kerstens PJ, Hazes JM, et al. Clinical and radiographic outcomes of four different treatment strategies in patients with early rheumatoid arthritis (the BeSt study): A randomized, controlled trial. Arthritis Rheum 2005;52:3381-90.

27. Lati C, Guthrie LC, Ward MM. Comparison of the construct validity and sensitivity to change of the visual analog scale and a modified rating scale as measures of patient global assessment in rheumatoid arthritis. J Rheumatol 2010;37:717-22.

28. Cella D, Yount S, Sorensen M, Chartash E, Sengupta N, Grober J. Validation of the Functional Assessment of Chronic Illness Therapy Fatigue scale relative to other instrumentation in patients with rheumatoid arthritis. J Rheumatol 2005;32:811-9.

29. Yount S, Sorensen MV, Cella D, Sengupta N, Grober J, Chartash EK. Adalimumab plus methotrexate or standard therapy is more effective than methotrexate or standard therapies alone in the treatment of fatigue in patients with active, inadequately treated rheumatoid arthritis. Clin Exp Rheumatol 2007;25:838-46.

30. Wells G, Li T, Maxwell L, Maclean R, Tugwell P. Responsiveness of patient reported outcomes including fatigue, sleep quality, activity limitation, and quality of life following treatment with abatacept for rheumatoid arthritis. Ann Rheum Dis 2008;67:260-5.

31. Krishnan E, Sokka T, Häkkinen A, Hubert H, Hannonen P. Normative values for the Health Assessment Questionnaire Disability Index: Benchmarking disability in the general population. Arthritis Rheum 2004;50:953-60

32. Ware JE, Kosinski M. SF-36 Physical and Mental Health Summary Scales: a manual for users of version 1, second edition. Lincoln, RI: QualityMetric Incorporated; 2001:28-35.

33. Russell AS. Quality-of-life assessment in rheumatoid arthritis. Pharmacoeconomics 2008;26:831-46.

34. Strand V, Bombardier C, Maetzel A, Scott D, Crawford B. Use of minimum clinically important differences [MCID] in evaluating patient responses to treatment of RA [abstract]. Arthritis Rheum 2001;44 Suppl:S187.

35. Zhao SZ, Fiechtner JI, Tindall EA, Dedhiya SD, Zhao WW, Osterhaus JT, et al. Evaluation of health-related quality of life of rheumatoid arthritis patients treated with celecoxib. Arthritis Care Res 2000;13:112-21.

36. Marra CA, Woolcott JC, Kopec JA, Shojania K, Offer R, Brazier JE, et al. A comparison of generic, indirect utility measures (the HUI2, HUI3, SF-6D, and the EQ-5D) and disease-specific instruments (the RAQoL and the HAQ) in rheumatoid arthritis. Soc Sci Med 2005;60:1571-82.

37. Walters SJ, Brazier JE. What is the relationship between the minimally important difference and health state utility values? The case of the SF-6D. Health Qual Life Outcomes 2003;1:4.

38. Harrison MJ, Davies LM, Bansback NJ, Ingram M, Anis AH, Symmons DP. The validity and responsiveness of generic utility measures in rheumatoid arthritis: A review. J Rheumatol 2008;35:592-602.

39. Feeny DH, Torrance GW, Furlong WJ. Health Utilities Index. In: Spilker B, ed. Quality of life and pharmacoeconomics in clinical trials. 2nd ed. Philadelphia: Lippincott-Raven Press; 1996:239-52.

40. Horsman J, Furlong W, Feeny D, Torrance G. The Health Utilities Index $\left(\mathrm{HUI}^{\circledR}\right)$ : Concepts, measurement properties and applications. Health Qual Life Outcomes 2003;1:54.

41. Furlong WJ, Feeny DH, Torrance GW, Barr RD. The Health Utilities Index (HUI) system for assessing health-related quality of life in clinical studies. Ann Med 2001;33:375-84.

42. Fairclough DL. Design and analysis of quality of life studies in clinical trials. Boca Raton, FL: Chapman \& Hall/CRC; 2002:153-81.

43. Strand V, Crawford B, Singh J, Choy E, Smolen JS, Khanna D. Use of "spydergrams" to present and interpret health-related quality of life data across rheumatic diseases. Ann Rheum Dis 2009;68:1800-4. 